\title{
Abraham dans le texte coranique : Itinéraire vers la convergence universelle
}

\author{
Majida Sayegh, \\ Université Libanaise, Faculté des Lettres et des Sciences Humaines \\ (Section 5) Département de Langue et Littérature Françaises, Lebanon
}

Doi:10.19044/esj.2020.v16n17p80 ～URL:http://dx.doi.org/10.19044/esj.2020.v16n17p80

\section{Résumé}

Basée sur le texte coranique, l'étude vise à examiner le trajet d'Abraham jalonné par ses observations rigoureuses et sa lutte contre le paganisme pour aboutir au socle du monothéisme. La façon de confronter l'idolâtrie de son peuple montre qu'Abraham a été le fondement d'une approche intellectuelle basée sur la raison et la réflexion. Ses luttes sur les différents fronts ne sont qu'une confirmation de cette démarche, un point de départ pour raser le bourbier de la superstition et d'aller de l'avant sur les plans individuel et universel.

Mots-clés : Paganisme, monothéisme, pensée, raison, universel 


\title{
Abraham in the Quranic Text: Route Towards Universal Convergence
}

\author{
Majida Sayegh, \\ Université Libanaise, Faculté des Lettres et des Sciences Humaines \\ (Section 5) Département de Langue et Littérature Françaises, Lebanon
}

\begin{abstract}
Based on the Koranic text, the study aims to examine Abraham's path marked by his rigorous observations and his struggle against the paganism to achieve the foundation of monotheism. The way of confront the idolatry of his people shows that Abraham was the foundation of an intellectual approach based on reason and reflection. His struggles on the various fronts are only a confirmation of that path, a starting point to raze the quagmire of superstition and move forward on the individual and universal levels.
\end{abstract}

Keywords: Paganism, monotheism, thought, reason, universal

\section{Introduction}

Chaque texte dispose d'un certain pouvoir informatif qui exige de la part du destinataire une capacité de réception. Dans cette optique, le texte du Coran qui se distingue par sa brièveté et son laconisme met le lecteur dans une position de réflexion et de contemplation, surtout quand le même thème est traité à plusieurs reprises dans de différentes sourates (chapitres).

Le Coran, qui date de plus de 1400 ans, s'adresse avant tout à l'être humain en tant qu'individu, quelle que soit sa race ou sa religion : bien qu'il soit en (Les Poètes, 195) «claire langue arabe » qui charme plus d'un arabophone. Le Clézio, Prix Nobel de la littérature 2008, est séduit par cette « langue-musique [...] qui s'enroule autour de toi et va jusqu'à ton cœur » (Barbey et al., 2003, p.138). Pourtant, ce n'est pas l'aspect poétique de la langue arabe qui est visé comme champ d'étude dans cet article mais le contenu des versets coraniques à l'aide de la traduction française.

L'itinéraire d'Abraham à travers le Coran se veut sans préjugés, loin des vues confessionnelles et religieuses étroites. L'approche objective et analytique avec le texte vise avant tout à explorer l'ampleur informative des versets examinés en insistant sur la signification des mots qui « ne décrivent en vérité que leur propre pouvoir » comme le dit (Zarai, 2012). L'aspect littéral de l'énoncé sera parfaitement respecté : le but est d'en extraire sa 
dimension humaine surpassant les frontières spatiales et temporelles. La démarche de l'analyse sera guidée par la logique qui « se clôt sur elle-même, réseau de relations purement externes, totalement indépendant de son contenu » selon (De Visscher, 1985). Dans cet esprit, cette recherche envisage le rôle d'Abraham en tant que père des prophètes monothéistes : le but n'est pas de raconter sa vie, car le Coran, et comme le dit si bien (De Prémare, 2006) « n'est pas un livre d'histoire » ni « un récit historique », mais de comprendre son cheminement intellectuel qui a abouti à la conception de l'unicité de Dieu. A cet effet, « aucun aspect de l'analyse des éléments du langage ne doit pas être abandonné » (Aziegbe, 2019).

L'étude moderne du Coran réalisée par le penseur Muhammad Shahrour préconise de traiter le texte coranique comme s'il avait été révélé hier « read the Qur'an as if it was revealed last night», en affirmant une approche universaliste basée sur la convergence et non sur la divergence entre toutes les confessions du monde. Selon son ouvrage philosophique (Shahrur, 2009), les Musulmans (Al-muslimūn) sont ceux qui se soumettent à un Créateur Unique, c'est la religion d'Abraham, un arbre de trois branches : Judaïsme, Christianisme et Islam. Leurs adeptes sont tous croyants en Dieu Unique (La Vache, 62) «...quiconque croit en Dieu et au Jour dernier et pratique le bien, ceux-là trouveront leur récompense auprès de leur Seigneur et ils n'éprouveront ni crainte, ni affliction ». Il est bien connu qu'Abraham est un prophète rassembleur, à travers lui, se rencontrent les trois religions monothéistes. Les Juifs, les Chrétiens et les Musulmans représentent ensemble environ $54.8 \%$ de la population mondiale en 2010 et $56.2 \%$ un pourcentage estimé pour 2020 (Hackett, Connor, Stonawski, \& Vegard, 2015).

Réellement, le mot "Abraham" a été mentionné dans le Coran 68 fois, en plus de la sourate portant son nom, et contenant 52 versets où il est présenté comme (Les femmes, 125) «Un pur croyant, que Dieu a pris pour ami », en l'honneur de son dévouement et de son sacrifice. Il est à noter que (125) désigne le numéro du verset dans la sourate, les mêmes notations ont été adoptées dans l'ensemble du papier.

Le site (Electronic Qur'an , www.E-Quran.com, 2009) a été utilisé pour la version française du texte coranique sachant que ce site contient des traductions du Coran en 58 langues différentes. Les sourates qui constituent notre corpus sont: (La Vache, 62,124,128,130,143,258,260), (Marie, 42,43,46), (Houd, 75), (L'Abeille, 93,120), (Le Bétail, 6,76-79), (Les Femmes, 125), (Les Prophètes, 52-56,58-61,63-64,66-69,73), et enfin (Les Rangs, 8889,91-96,102,104-105,107,109).

Ce papier est divisé en cinq sections de recherche : la première section est l'introduction elle-même. La seconde section étudiera la capacité logique d'Abraham comme un témoin éloquent de la recherche de l'unicité de Dieu, tandis que la troisième section sera consacrée à l'itinéraire d'Abraham pendant 
la propagation de sa nouvelle croyance. Dans la quatrième section, l'accent sera mis sur les principaux facteurs qui ont consacré Abraham comme le père du monothéisme et un modèle pour l'humanité. Enfin la cinquième section sera réservée à la conclusion.

\section{Abraham et la recherche de l'existence et de l'unicité de Dieu}

Abraham a utilisé une logique simple et incontournable pour illustrer la grandeur et l'unicité de Dieu, et cette situation s'est traduite par un certain nombre de versets qui seront étudiés au fur et à mesure. Le Coran a mentionné dans les sourates «Le Bétail » et «Les Rangs », comment Abraham est parvenu par sa pensée rationnelle à la conviction en prouvant la validité d'une hypothèse par le rejet de sa contradiction. Cette approche a pour but d'examiner les versets coraniques concernés dans lesquels sont illustrées les épreuves d'Abraham durant son cheminement douloureux vers l'unicité de Dieu. Dans cette intention, il eut recours à la contemplation attentive du ciel plein de secrets en frayant un chemin vers la déduction de l'existence et de l'unicité du Créateur (Les Rangs, 88) «Puis il regarda attentivement les étoiles ».

Vivant dans le doute, Abraham était inquiet et perplexe à en devenir malade dans le sens psychologique du terme (Les Rangs, 89) «Je suis vraiment malade ». Sa conscience religieuse qui cherchait ardemment la vérité se formait graduellement : une attitude allant en harmonie avec le fond du Coran. C'est ce que (Sayegh M. , 2016) confirme dans son article « le Coran met l'accent sur la germination des choses et des créatures et précise l'accès au savoir au moyen de l'apprentissage et que ce savoir est gradué et jamais il n'arrive d'un seul coup et que tout homme est capable d'y accéder, s'il le désire et le cherche ».

En effet, les circonstances temporelles dans lesquelles Abraham suivait ses contemplations sont essentielles : au crépuscule (Le Bétail, 76) «Lorsque la nuit l'enveloppa, il vit l'étoile ». Ces moments sont propices à la rêverie et la méditation profonde. Scrutant de ses yeux, assoiffés de vérité, les étoiles brillantes innombrables qui décorent et magnifient le ciel avec magie, Abraham fut attiré par une planète lumineuse: serait-elle Venus ? Effectivement, elle est proche de la terre et l'observateur peut la voir en permanence en l'absence de pluie et de brouillards. Pour cela, le verset dit, "il a aperçu", donc il a vu par ses propres yeux, il ne lui a pas donné le caractère de l'émergence. Cela signifie que l'état de la planète était comme dans l'immuabilité pour les observateurs et elle ne se déplace pas comme la lune ou le mouvement apparent du soleil (Le Bétail, 76) «Abraham dit : Ceci est mon Seigneur !». Mais après une contemplation prolongée dans le temps et la constatation de sa disparition, Abraham se posa la question : - Cette étoile brillante serait-elle Dieu ? Et il se répond (Le Bétail, 76) « Je n'aime pas ceux 
qui disparaissent ». Abraham continue sa méditation dans le ciel (Le Bétail, 77) «Lorsqu'il vit la lune se lever », alors il fut attiré par une comparaison ciblée et attractive entre la lumière de la planète qui semble faible et le clair de lune scintillant dans le ciel, «coulant comme une cascade au cour de l'horizon » comme le dit (Fadlallah, M,H, 1998, Vol. 9, p.180), ce qui a poussé Abraham à déclarer vite (Le Bétail, 77) « Ceci est mon Seigneur ! ». Mais son opinion n'a pas pu se maintenir longtemps car le clair de la lune n'a pas duré et il a commencé à s'estomper. Ainsi Abraham retombe dans la perplexité en se demandant où est Dieu et où est-Il parti ? Est-ce que le véritable Dieu s'absente-t-Il et disparait-Il ? Cette lutte sans fin entre l'aberrance et le bon sens a conduit Abraham à poser des questions profondes concernant Dieu : Comment Dieu est ? Où est-Il ? Et ce qui l'a conduit à prier la Toute-Puissance afin de le sauver des tourments de l'égarement (Le Bétail, 77) «Il dit : Si mon Seigneur ne me guide pas, je serai parmi les égarés ».

Désespéré par les planètes et la lune, Abraham déplace son attention sur la force et la grandeur du soleil qui contrastent fortement avec les deux exemples précédents (Le Bétail, 78) «Ceci est mon Seigneur, ceci est plus grand ! ». Pourtant sa déception ne tarde pas à s'installer après le coucher du soleil laissant la terre dans le froid et l'obscurité. La grandeur matérielle s'avère inutile dans la caractérisation de Dieu dont la gloire et la toutepuissance ne seraient comparées à aucun objet perçu quelle que soit sa taille ou sa force. Ainsi, l'idée de l'essence divine se développe et se purifie graduellement dans l'esprit d'Abraham chez qui la notion du Dieu immatériel commence à naître. En réalité, d'après l'expérience observatrice des planètes et des étoiles, Abraham a pu constater que le vrai Dieu ne peut pas changer ou subir des transformations à l'instar des créatures existantes parce qu'Il est le Créateur Lui-même et il se distingue par l'immuabilité qui est l'un des attributs divins.

Après ce grand réveil intellectuel et spirituel, Abraham cria à son peuple (Le Bétail, 78) «O mon peuple ! Je suis innocent de ce que vous associez à Dieu ». En déclarant son innocence des superstitions de son peuple, il se rebelle contre le polythéisme de son époque qui rabaissait la notion de la divinité. Accédant à cette constatation de base, Abraham ressent le besoin de percer le secret de la vie et de la mort : désir suprême de tout être humain tourmenté par sa finitude et ses limites. A cet effet, le dialogue avec le Seigneur vise à renforcer la foi en Lui. C'est pourquoi Abraham a demandé à Dieu de lui montrer comment ressusciter les morts (La Vache, 260) «Mon Seigneur ! Montre-moi comment tu fais revivre les morts ». Il voulait observer la résurrection, une expérimentation qui effacerait le moindre doute (La Vache, 260) «Dieu dit : Est-ce que tu ne crois pas ? Il répondit : Si, assurément, mais c'est pour que mon cœur soit apaisé ! ». La réponse divine était une question rhétorique et la réponse d'Abraham était une affirmation de 
sa foi, mais sa demande visait à gagner plus d'assurance et d'apaisement intérieurs.

Finalement, Dieu a réalisé le désir d'Abraham en lui demandant de participer lui-même à l'expérience pour consolider sa foi en la résurrection (La Vache, 260) «Dieu dit : Prends quatre oiseaux ; apprivoise-les pour qu'ils reviennent vers toi, puis sacrifie-les et place chacun d'eux sur une des montagnes environnantes ; ensuite, appelle-les : ils accourent vers toi promptement. Sache que Dieu est omnipotent et sage ! ». La démonstration des quatre oiseaux ressuscités comble l'esprit d'Abraham de certitude. C'est une confirmation de la nécessité chez l'homme d'observer, d'analyser puis de déduire.

Après ce voyage réflexive dans la connaissance de Dieu, Abraham constate qu'on ne peut pas sentir Dieu comme on sent la présence des objets et des êtres créés à partir des cinq sens ; il se rend compte que toutes les créatures physiques et vivantes dans ce grand univers ne sont que des réalisations de la toute-puissance de Dieu et déclare (Le Bétail, 79) «Je tourne mon visage, en pur croyant, vers Celui qui a créé les cieux et la terre, et je ne suis pas parmi les associateurs ».

\section{Abraham et la propagation de la nouvelle croyance}

Dans la première section, nous avons démontré comment Abraham a délibérément pris la voie de la contemplation méditative du ciel dans le but de rechercher le Seigneur. L'intérêt qu'il a porté aux planètes et aux étoiles signale l'interférence de la terre et du ciel. Effectivement, le globe terrestre vit en symbiose avec le reste des planètes selon des lois physiques reconnues exprimées à travers des phénomènes comme la pluie et l'alternance du jour et de la nuit. Ainsi, l'idée de Dieu, Créateur et Maître de la vie, était fortement présente dans son esprit et dans toute son entité spirituelle. Après cette recherche assidue de Dieu et une fois Abraham acquit la conscience et la certitude de son existence, une phase avancée s'impose : la propagation de la nouvelle croyance basée sur l'unicité de Dieu immatériel. Il a commencé son dialogue avec les gens les plus proches de lui. Le fait de débuter le dialogue avec son père est porteur d'un sens profond: se rebeller contre le père, c'est se révolter contre une partie de sa propre personnalité malgré le lien naturel qui unit le père et le fils. C'est le complément incontournable du travail intellectuel mené sur lui-même dans la phase précédente (Marie, 42) «Il dit à son père : $\mathrm{O}$ mon père ! Pourquoi adores-tu ce qui n'entend pas, ne voit pas, et ne t'est d'aucun profit ?». Une question qui renferme implicitement sa propre réponse : la caractérisation négative de l'idole est frappante et n'attend aucune réponse.

Abraham poursuit son argumentation en invitant son père à le suivre (Marie, 43) « O mon père ! Il m'est échu une portion de la science qui ne t'est 
pas parvenue. Suis-moi : je te guiderai sur une voie droite ». Ce verset éclaire un élément fondamental de la démarche d'Abraham comme un leadership qui détient la connaissance suffisante et mérite d'être suivi par les autres parce qu'il est capable de les guider vers le discernement. La raison avancée par Abraham est la possession de la science en tant que faculté maitresse qui arme l'être humain et le distingue de ses semblables. Il jugeait que l'héritage des anciens comme une abomination qui ne menait qu'à l'erreur et l'aliénation. C'est ce qu'affirme (Fadlallah, M,H, 1998, Vol. 15, p.51-52) « Il ne s'agit pas de père ou de fils, c'est une question d'ignorant et de connaisseur. Pas une histoire de longue ou de courte durée de la vie d'une personne parce que l'importance de l'âge est dans l'expérience vécue et non pas dans les moments chronophages ». Le père réagit négativement et demande sans détour à son fils de s'éloigner définitivement (Marie, 46) «Il dit : O Abraham, éprouverais-tu de l'aversion pour mes divinités ? Si tu ne cesses pas, je te lapiderai. Éloignetoi de moi pour un temps ». Ce dialogue échoué reflète l'incapacité des anciens accablés par l'immobilisme à suivre les idées avancées de la nouvelle génération. Cela concrétise la rupture inévitable et fondamentale spécifique de toute révolution ce qui va dans la direction de l'évolution de l'humanité.

Ensuite, Abraham se retourne vers les masses de sa communauté pour dialoguer avec elles : (Les Rangs, 91) « Il se glissa alors vers leurs divinités et dit : Quoi donc, vous ne mangez pas ?», (Les Rangs, 92) « Pourquoi ne parlez-vous pas ? », (Les Rangs, 93) «Furtivement, il les frappa de sa main droite », (Les Rangs, 94) «Les gens accoururent vers lui précipitamment », (Les Rangs, 95) «Adorez-vous ce que vous avez sculpté, leur dit-il » et (Les Rangs, 96) «alors que c'est Dieu qui vous a créés, vous et les œuvres de vos mains ? » Ces versets informent qu'Abraham se moquait de leurs idoles en les qualifiant de pierres sculptées, sourdes et muettes. Il affirme à son peuple que c'est Dieu qui a créé les êtres humains et les œuvres qui pourraient sortir de leurs mains. En se basant sur l'idée selon laquelle on ne peut pas adorer un objet que nous avons fabriqué nous-mêmes (Les Prophètes, 52) «Il dit à son père et à son peuple : Que sont ces statues à qui vous rendez un culte ? ». Leur réponse était (Les Prophètes, 53) « Nous avons trouvé nos pères voués à leur adoration ». Abraham les accuse, eux et leurs pères, par (Les Prophètes, 54) "un égarement manifeste". En effet, il leur explique qu'ils ne peuvent pas hériter une foi égarée prétendument religieux qui s'est transformée en une tradition aveugle où aucun rôle n'est accordé à l'esprit et au raisonnement.

Le peuple qui était très réticent qualifie Abraham de plaisantin qui doit présenter ses excuses sous prétexte de son mépris envers les idoles (Les Prophètes, 55). Mais il le déçoit en affirmant que Dieu est le seul Créateur de cet univers avec tout ce qu'il contient. Et il affirme que cette conviction est enracinée en lui et dans son comportement (Les Prophètes, 56) « Il dit : Tout au contraire ! Votre Seigneur est le Seigneur des cieux et de la terre ; c'est Lui 
qui les a créés, et moi j'en porte témoignage ». Ainsi, Abraham se démarque de son peuple qui refuse toute réceptivité et une confrontation est annoncée. Pourtant il change de stratégie. Il se rend compte qu'il est en face des masses ignorantes et rétrogrades qui ont besoin d'autres illustrations plus concrètes. C'est pourquoi il a eu l'idée de fracasser leurs idoles (Les Prophètes, 58) « Il les mit en pièces, à l'exception de la plus grande, afin qu'ils puissent se tourner vers elle » et ceci pour s'en servir dans son raisonnement ultérieur. En effet, cette destruction ne résulte pas d'une colère ou d'une vengeance mais sert à son argumentation devant le rassemblement païen. En réalité, après la découverte de cette démolition, le peuple demande à Abraham de témoigner (Les Prophètes, 59-61) «Ils dirent : Celui qui a agi ainsi avec nos dieux est vraiment inique !», « Certains dirent : Nous avons entendu un jeune homme qui les mentionnait; il se nomme Abraham » et «Ils dirent : Amenez-le donc sous les yeux des gens pour qu'ils puissent témoigner ». Mais sa réponse était déroutante: (Les Prophètes, 63) «Il dit : Mais non ! C'est celui-là, le plus grand d'entre eux, qui l'a fait. Interrogez-les donc, s'ils peuvent parler !». Il voulait par sa réponse générer un choc intellectuel qui ébranle leurs convictions à ce sujet. C'est là que se distingue la ruse logique d'Abraham : il ne voulait pas mentir à propos de la grande idole mais il avait pour objectif d'ouvrir la voie au raisonnement, c'est ce que confirme (Fadlallah, M,H, 1996, p.91).

Cette stratégie d'Abraham a bien fonctionné car ils revenaient à euxmêmes (Les Prophètes, 64) «S'étant ravisés, certains dirent : C'est vous qui êtes les iniques !». Mais la majorité, et malgré sa faiblesse devant l'argument d'Abraham, déclare que les idoles sont incapables de parler. Alors il leur répond: (Les Prophètes, 66) «Adorez-vous donc, en dehors de Dieu, ce qui ne peut ni vous être utile en quoi que ce soit, ni vous nuire ?». Abraham se plaint de la réaction négative de son peuple en annonçant ouvertement (Les Prophètes, 67) « Honte à vous et à ce que vous adorez en dehors de Dieu ! Ne réfléchissez-vous pas ? ». Après ce discours audacieux d'Abraham, les gens se sont levés contre lui pour soutenir leurs idoles et décidèrent de le jeter dans le feu (Les Prophètes, 68) «Ils dirent : Brûlez-le ! et secourez vos dieux, si vous en êtes capables !». Mais le Seigneur a rendu le feu sans effet sur lui (Les Prophètes, 69) « Nous dîmes : O feu ! Sois, pour Abraham, fraîcheur et paix ! ». Par ce miracle, Dieu voulait démontrer sa toute-puissance devant les païens et insister sur la place privilégiée d'Abraham auprès de Lui.

Sachant l'importance du rôle des souverains dans les destins des peuples, Abraham est présenté par le texte coranique comme cherchant les sources des maux de l'humanité parmi lesquels la tyrannie. En effet, le Coran se réfère à un débat entre Abraham et un tyran dont le nom n'est pas cité, un souverain imbu de soi-même et de sentiment de grandeur. Afin de le raisonner, Abraham lui explique que Dieu est celui qui détient le pouvoir absolu (La 
Vache, 258) «Abraham ayant dit : Mon Seigneur est Celui qui fait vivre et qui fait mourir ». Le tyran répliqua «Moi, je fais vivre et je fais mourir » par conséquent les gens doivent l'adorer car il a le pouvoir de sauver un condamné à mort ou de l'exécuter. Alors, il serait le maître de la vie et de la mort. C'est à ce moment-là qu'Abraham fait référence aux mouvements cosmiques pour réfuter l'argument du tyran en lui montrant les limites de ses pouvoirs devant la toute-puissance divine (La Vache, 258) « Dieu fait venir le soleil de l'Orient ; fais-le donc venir de l'Occident !». Le tyran fut désarmé devant un tel argument.

Le dialogue se révèle comme une stratégie adoptée par Abraham avec tous ses détracteurs. En poursuivant cette approche calme et raisonnée, il visait la persuasion rationnelle qui se base sur les observations et la déduction, pour combattre la superstition et ses origines.

\section{Abraham père spirituel du monothéisme et modèle pour les hommes}

Pour les trois religions, le Judaïsme, le Christianisme et l'Islam, «Abraham symbolise le principe fondateur de leurs religions respectives » comme indique (Guiton, 2004). Pareillement, Ernest Renan dans son livre, (Renan, 1992[1857,86]) écrit que «ces trois grands mouvements religieux sont trois faits sémitiques, trois rameaux du même tronc, trois traductions inégalement belles de la même idée ». Aussi d'autres religions se mettent-elles en concordance avec les principes universels de son message à l'homme indépendamment de sa race ou de son ethnie. N'est-il pas celui qui est désigné comme un modèle par son Dieu ? Abraham peut être considéré comme un arbitre sur les points de différences entre les disciples des prophètes comme dit (Fadlallah, M,H, 1996, p.8).

Dans le verset (La Vache, 124), l'accent est mis sur l'épreuve d'Abraham dans son mouvement du polythéisme au hanafisme, c'était un détournement décisif de l'idolâtrie vers un Créateur unique, une marche pleine d'enseignements et de leçons pour construire le fond humain ambitieux plein de paix et d'ouverture. Comme nous l'avons vu dans les deux sections précédentes, Abraham a traversé des expériences qui lui ont permis d'atteindre sa foi dans l'unicité du Créateur et de combattre les concepts religieux rétrogrades de son époque. La réussite dans toutes les épreuves à la fois théoriques et pratiques contribua à parfaire ses caractéristiques en tant que leader (La Vache, 124) «Dieu lui dit : Je vais faire de toi un modèle pour les hommes ». Étant donné l'importance de la mission que Dieu lui confie, Abraham prend conscience de son rôle en tant que modèle. Dieu lui procure des enfants malgré son âge avancé, afin de perpétuer ce rôle dans les générations futures (La Vache, 124) «Et quoi de ma descendance ? demanda Abraham ». Cela reflète sa conscience du mouvement de l'histoire illimitée à 
travers le temps et l'espace : les différentes communautés ont besoin de leurs leaders respectifs aptes à les guider selon les principes de la convergence universelle. Ce désir de guider n'a rien à voir avec les privilèges ou les honneurs personnels où le pouvoir est transmis de père en fils pour des raisons égoïstes comme l'affirme (Fadlallah, M,H, 1998,Vol. 3,p.12). Le véritable but est de faire régner la foi. (La Vache, 124) «Le Seigneur répondit : Mon alliance ne s'étend pas aux iniques », autrement dit, ce n'est pas un droit de faire hériter à un fils qui régnerait dans l'injustice, l'oppression et la transgression. Cette déduction ouvre la voie à la gouvernance du pouvoir pour valoriser la liberté et la justice. Ceci est confirmé par le verset (La Vache, 128) «Notre Seigneur! Fais de nous deux des croyants qui Te soient soumis; et fais de notre descendance une communauté qui Te soit soumise »: Abraham prie son Seigneur de faire de lui et de ses descendants, des soumis à Dieu dans leurs paroles, leurs actions, leurs comportements.

Le fait d'insister sur l'adoration unique pour Dieu ouvre la voie à la dignité et la liberté pour les êtres humains qui ne doivent pas être esclaves les uns des autres. Ainsi, la religion d'Abraham vise à libérer l'homme des oppressions exercées non seulement par la superstition et l'idolâtrie, mais aussi par les hommes tyranniques. Ainsi, la notion de l'unicité de Dieu est une pensée salvatrice au niveau humain, (La Vache, 130) «Qui donc ressent de l'aversion pour la religion d'Abraham, sinon celui qui s'égare dans la folie ?». Le message de l'obéissance à Dieu libère des autres soumissions humiliantes et aliénantes pour l'homme. C'est pourquoi Abraham est le modèle (L'abeille, 120) «En vérité, Abraham fut un modèle de soumission à Dieu ». Le mot "modèle" est employé dans le sens du bon commandant qui guide les gens dans la bonne voie pour le bien de l'humanité entière : (Les Prophètes, 73) «Nous en avons fait des modèles (imâms) qui guident selon Notre Commandement, et Nous leur avons révélé la pratique du bien, l'accomplissement de la prière et le don de l'aumône. Pour Nous, ils étaient des serviteurs dévots ». A travers ce rôle de commandant, Abraham est consacré comme le patriarche de toute la lignée des prophètes monothéistes.

Le texte coranique insiste sur le tronc commun unissant les trois religions monothéistes : la diversité des messages divins du Judaïsme, du Christianisme et de l'Islam n'est au fond qu'une manifestation diversifiée du message d'Abraham, père du monothéisme : (L'Abeille, 93) « Si Dieu l'avait voulu, Il aurait fait de vous une communauté unique... ». Le rôle qui incombe à cette communauté monothéiste visée par le texte coranique est grand (La Vache, 143) «Ainsi, Nous avons fait de vous une Communauté du juste milieu pour que vous soyez témoins envers les hommes ». L'extrémisme n'y existe pas, le centrisme ici n'est pas une situation géographique mais il s'agit d'une conviction basée sur la modération, le respect et la tolérance. Dans ce 
contexte, il est important de souligner que le mot " soumission" ou "Islam" et les mots qui en dérivent ont été mentionnés 140 fois dans le Coran.

L'exemplarité d'Abraham en tant que commandant et modèle s'est couronnée avec l'épreuve de la tentation de sacrifier son fils, phase ultime de son témoignage de fidélité envers Dieu. Ce récit de sacrifice « peut-être le récit le plus dramatique et le plus émouvant du cycle patriarcal »comme le dit si bien (Berthoud, 2006). Un épisode qui comporte une leçon divine qui vise deux objectifs : d'une part, la foi et la soumission totale d'Abraham à Dieu et d'autre part le fait de vivre les horreurs de cette tentative aide les générations futures à la rejeter.

Abraham est donné en exemple vivant de la confiance totale en Dieu. Son fils participe à cette épreuve car il est en âge de discuter avec son père et présente également un autre exemple de foi et de docilité (Les Rangs, 102) «Lorsque celui-ci fut parvenu à l'adolescence, son père lui dit : O mon fils ! J'ai fait un songe où j'ai vu que je t'immolais : vois, qu'en penses-tu ?» En réalité, les songes ont joué un rôle déterminant dans le monothéisme, comme elle l'affirme (Sayegh, M, 2019), mais si la vision de Joseph était le point de départ d'un itinéraire prophétique par excellence, celle d'Abraham constitue au contraire un point culminant de son cheminement et scelle une amitié horspair avec Dieu. Ce qui attire fortement l'attention est la réponse du fils à Abraham (Les Rangs, 102) «O mon père ! Fais ce qui t'est ordonné. Si Dieu le veut, tu me trouveras parmi ceux qui sont patients !». Il est conscient du rôle de son père et de son assujettissement complet à son Créateur ; il sera patient car il possède la même foi. Il est bien connu que les religions païennes pratiquaient l'immolation des humains comme une offrande à leurs divinités. C'était le crime le plus abominable commis par des parents envers leurs enfants. Et quand Abraham a mis son fils sur son front à terre, Dieu l'a appelé (Les Rangs, 104) « Nous lui criâmes : O Abraham! », (Les Rangs, 105) « Tu as vraiment cru en cette vision. C'est ainsi que Nous récompensons les hommes de bien ». Dieu lui a fait descendre un grand bélier en échange de son fils (Les Rangs, 107) «Nous avons racheté son fils par une généreuse immolation ».

Il ressort clairement à quel point l'épreuve d'Abraham était terrible, ainsi que celle de son fils, et comment le père et son fils ont été entièrement fidèles à Dieu. En étant (Houd, 75) « charitable, humble et repentant », Abraham représente la foi inébranlable du monothéisme (Les Rangs, 109) «Que la Paix soit sur Abraham!».

\section{Conclusion}

Le texte coranique affirme que le cheminement d'Abraham vers le monothéisme fut guidé par le principe de dialogue sous ses différentes formes : le dialogue avec lui-même, le dialogue avec Dieu, puis avec son père, 
avec son peuple et ses souverains. Ces dialogues fondés sur la raison et la logique cherchaient la vérité divine dans un mouvement méthodique et ascendant. En effet, les épreuves étaient d'abord inhérentes à l'esprit et à l'âme d'Abraham qui luttait contre ses propres doutes dans un tâtonnement évolutif qui résumerait le cheminement de la raison humaine. Une fois l'assurance et la certitude acquises, il entame la période de la propagation de la foi nouvelle qui fut épuisante car il luttait contre trois ennemis également farouches et hostiles à tout changement : premièrement l'ancienne génération représentée par le père, deuxièmement l'ignorance et le fanatisme à travers le peuple et enfin le pouvoir tyrannique gardien de l'obscurantisme régnant.

Avec l'épreuve de la tentation du sacrifice de son fils, Abraham sanctifie cette alliance particulière avec son Créateur et met un terme au rite le plus horrible de l'histoire de l'humanité : l'immolation des êtres humains. Abraham a vécu des expériences à la fois méditatives et pratiques suffisantes pour le consacrer comme un modèle qui guide vers la voie du monothéisme constructive pour toute l'humanité. Il est présenté par le texte coranique comme un «monde » et un « juste milieu » qui unifient, non seulement les trois religions monothéistes, mais tout le genre humain autour de ses principes : dialogue qui répugne à toute violence, principes de miséricorde universels et foi inébranlable en Dieu unique. Ce qui incite à lutter contre le fanatisme sous ses différentes formes pour le bien de l'humanité. C'est dans ce sens que l'expression «L'Ami de Dieu » qualification coranique exclusive à l'égard d'Abraham, prend toute sa valeur.

\section{References:}

1. Aziegbe, M. E. (2019, February). Analyse De L'unité Morphologique Comme Signe Linguistique À Double Face Destinée Aux Apprenants Universitaires Au Nigéria : Le Cas De La Flexion Verbale En Français. European Scientific Journal, 236-253.

2. Barbey, B.; Le Clézio, J.M.G.; Le Clézio, J. (2003). Maroc. Éditions de la Martinière.

3. Berthoud, P. (2006). La mise à l'épreuve décisive d'Abraham (Genèse 22.1-19), théologie évangélique (ThEv), 5(3), 209-232.

4. De Prémare, A.-L. (2006). Le Coran, enquête sur un livre sacré. 1-7. doi:https://www.lhistoire.fr/parution/collections-30.

5. De Visscher, L. F. (1985). Wittgenstein. Le langage à la racine de la question philosophique. Revue Philosophique de Louvain, 559-584.

6. Electronic Qur'an, www.E-Quran.com. (2009).

7. Fadlallah, M,H. (1996). Dialogue dans le Coran (éd. 5, Vol. 3). Dar AL Malak, Haret Hreik, Lebanon.

8. Fadlallah, M.H. (1998). Interprétation inspirée du Coran (éd. 2, Vol. 15). Dar AL Malak,Haret Hreik, Lebanon. 
9. Fadlallah, M.H. (1998). Interprétation inspirée du Coran (éd. 2, Vol. 9). Dar AL Malak, Haret Hreik, Lebanon.

10. Fadlallah, M.H. (1998). Interprétation inspirée du Coran (éd. 2, Vol. 3). Dar AL Malak, Haret Hreik, Lebanon.

11. Guiton, R. (2004). Abraham Littérature de l'imaginaire ? Revue Plaisance,(5). doi:https://www.rene-guitton.fr/conferences/abrahamlitterature-de-limaginaire

12. Hackett, C., Connor, P., Stonawski, M., \& Vegard, S. (2015). The Future of World Religions: Population Growth Projections, 20102050,. Pew Research Center,www.pewresearch.org.

13. Renan, E. (1992[1857,86]). Études d'histoire religieuse. Paris: Gallimard.

14. Sayegh, M. (2019). La sourate Joseph : Approche mytho-critique. Jordan Journal of Modern Languages and Literatures, 11(2), 111-126.

15. Sayegh, M. (2016). Considérations coraniques sur les mythes. Feuilles Universitaires, 40(1), 45-64.

16. Shahrur, M. (2009). The Qur'an, morality and critical reason : the Essential Muhammad Shahrur. LEIDEN - BOSTON : Translated, Edited, and with an introduction by Andreas Christmann.

17. Zarai, M. (2012). L'écriture du pouvoir et le pouvoir de l'écriture dans la littérature québécoise. Littératures. Université Michel de Montaigne - Bordeaux III, Thèse de Doctorat. doi:HAL Id: tel00759986 https://tel.archives-ouvertes.fr/ 